\title{
Trip distribution forecasting in fuzzy multi-commodity transportation networks
}

\author{
F. Zandi \\ Faculty of Engineering \& Technology, Alzahra University, Iran
}

\begin{abstract}
In this paper, the author models trip distribution in fuzzy multi-commodity transportation networks, and indicates its dimension consideration. First, current trip distribution is learnt by an approximation function for each commodity. Then, a quadratic model is used to calibrate these functions. The implementation of the proposed model is demonstrated through the use of a numerical example. Keywords: fuzzy, multi-commodity, approximation function, trip distribution.
\end{abstract}

\section{Introduction}

Trip distribution constitutes the second stage in the transportation planning process. Trip distribution models are used to determine the number of trips between each pair of zones when the number of trips generated/attracted by particular zones is known. Continual utilization of this model will enable future capital needs to be updated and identified. Generally, such information is essential to a variety of operational tasks; failure analysis to capacity planning and traffic engineering.

A survey of these models may be divided into the following types. In gravitybased models, regression techniques are applied to calibrate the parameters. The models are divided into linear $[1-3]$ and nonlinear $[4,5]$ regression models. The pioneers of these models are Willumsen [6] and Van Zuylen [7, 8]. Statistical models include the constrained generalized least squares model [9] and constrained maximum-Likelihood models [10-13]. Another group has been developed based on sort computing techniques such as fuzzy logic and neural network $[14,15]$. They compared their model based on multilayer perception neural network with maximum-likelihood doubly-constrained models. Then, Kalic and Teodorović [16] attempted to develop a technique for modeling trip 
distribution. The model developed represents the application of Genetic Algorithm (GA) for trip distribution forecasting. All the above-mentioned models forecast the trip distribution for single-commodity transportation networks.

Therefore, the basic research task in this article is to develop trip distribution forecasting from single-commodity to multi-commodity. This paper is organized into three sections. The second section contains the proposed method followed by a new method and dimension consideration of the problems in the case of large scale. A numerical example is given. Conclusions are presented in the third section.

\section{The proposed method}

Let us first define parameters of multi-commodity networks:

$T_{i j}^{k}=$ Number of interchanged trips of commodity $k$ between $i$ and $j$.

$P_{i}^{k}=$ Number of trips produced by commodity $k$ at zone $i$.

$A_{j}^{k}=$ Number of trips attracted by commodity $k$ at zone $j$.

$t_{i j}^{k}=$ Travel time of commodity $k$ between $i$ and $j$.

$L_{i j}^{k}=$ Optional adjustment factor for interchanges between zones $i$ and $j$ for commodity $k$.

$i=$ an origin zone, $j=$ a destination zone, $\hat{T}_{i j}^{k}=$ estimation of $T_{i j}^{k}$

Now, the proposed method is described as follows:

STEP 1 (normalize the parameters): using the linear scale transformation, the various scales are transformed into a comparable scale. Therefore, the parameters can be normalized:

$$
P_{i}^{k}(s)=\frac{P_{i}^{k}}{\max _{i} P_{i}^{k}}, A_{j}^{k}(s)=\frac{A_{j}^{k}}{\max _{j} A_{j}^{k}}, L_{i j}^{k}(s)=\frac{L_{i j}^{k}}{\max _{i, j} L_{i j}^{k}}, t_{i j}^{k}(s)=\frac{\min _{i, j} t_{i j}^{k}}{t_{i j}^{k}}
$$

If these parameters have trapezoid numbers, they can be converted to crisp numbers (2) by the Fuller method [17]:

$$
\widetilde{P}_{i}^{k}=\left(P_{i 1}^{k}, P_{i 2}^{k}, P_{i 3}^{k}, P_{i 4}^{k}\right) \quad P_{i}^{k}=\frac{1}{3}\left[P_{i 1}^{k}+P_{i 3}^{k}+\frac{1}{2}\left(P_{i 2}^{k}+P_{i 4}^{k}\right)\right]
$$

STEP 2 (Calculate approximation functions): our objective is to minimize the sum of squares errors for all pairs in $T_{i j}^{k}$ and $\hat{T}_{i j}^{k}$ : 


$$
\begin{gathered}
\hat{T}_{i j}^{k}=f_{k}\left(P_{i}^{k}(s), A_{j}^{k}(s), L_{i j}^{k}(s), t_{i j}^{k}(s)\right) \\
S S E=\frac{1}{2} \sum_{i=1}^{n} \sum_{j=1}^{m}\left[T_{i j}^{k}-f_{k}\left(P_{i}^{k}(s), A_{j}^{k}(s), L_{i j}^{k}(s), t_{i j}^{k}(s)\right)\right]^{2}
\end{gathered}
$$

Based on regularization, it is proven by the RBF neural network that this eqn is minimized by the approximation function $(5)$ : $[15,18]$

$$
\begin{array}{r}
\hat{T}_{i j}^{k}=\sum_{h=1}^{N} w_{h}^{k} G\left(\underline{X}_{i j}^{k}(s), \underline{X}_{[h]}^{k}(s)\right) \\
G\left(\underline{X}_{i j}^{k}(s), \underline{X}_{[h]}^{k}(s)\right)=e^{-\left(\left\|\underline{x}_{i j}^{k}(s)-\underline{x}_{[h]}^{k}(s)\right\|\right)^{2}} \\
\underline{X}_{i j}^{k}(s)=\left[P_{i}^{k}(s), A_{j}^{k}(s), L_{i j}^{k}(s), t_{i j}^{k}(s)\right]
\end{array}
$$

Also, eqn (5) can be expressed in matrix format:

$$
\underline{T}^{k}=G^{k} \cdot \underline{W}^{k}
$$

Therefore, the above approximation functions can be calculated by the weight vector (9):

$$
\underline{W^{k}}=\left(G^{k^{\prime}} G^{k}\right)^{-1} G^{k^{\prime}} \cdot \underline{T}^{k}
$$

STEP 3 (Calibrate the approximation functions): for calibrating the above approximation function, the proposed model $(\mathrm{P})$ is formulated, which is one of the main contributions of this paper.

A. Objective Functions 1: based on conservation of flow [19], the sum of trips produced for $k$-th commodity in zone $i$ should be equal to $P_{i}^{k}$ :

$$
\sum_{j=1}^{m} \hat{T}_{i j}^{k} \cong P_{i}^{k} \quad i=1, \ldots, n
$$

However, the sum of trips produced by commodity $k$ in zone $i$ may not be equal to $P_{i}^{k}$. Therefore, $\hat{T}_{i j}^{k}$ can be calibrated by coefficients $x_{i}^{k}, x_{j}^{k}$ :

$$
\sum_{j=1}^{m} \hat{T}_{i j}^{k} \cdot x_{i}^{k} \cdot x_{j}^{k} \cong P_{i}^{k}
$$

B. Objective Functions 2: similarly, the sum of trips attracted by commodity $k$ to zone should be equal to $A_{j}^{k}$ : 


$$
\sum_{i=1}^{n} \hat{T}_{i j}^{k} \cdot x_{i}^{k} \cdot x_{j}^{k} \cong A_{j}^{k}
$$

C. Objective Functions 3: also, the calculated trip-time frequency distribution for commodity $k$ should be equal to the trip-time frequency distribution of real trip distribution [20], i.e.,

$$
\sum_{t} \hat{T}_{i j}^{k} \cdot x_{i}^{k} \cdot x_{j}^{k}-\sum_{t} T_{i j}^{k} \cong 0
$$

D. Constraints: let $u_{i j}$ be the upper limit of the sum of all commodities in arc $(i$, j) of the multi-commodity network. Then:

$$
\sum_{k} \hat{T}_{i j}^{k} \cdot x_{i}^{k} \cdot x_{j}^{k} \leq u_{i j}
$$

The above objective functions and constraints construct the following mathematical programming. It should be mentioned that in Zimmerman [20], Hwang [22], Masud [23] and Murty [24] a fuzzy objective function has been converted to constraint linear programming. Now, we have utilized this idea to propose the following new model:

Find:

$$
x_{i}^{k}, x_{j}^{k}
$$

st:

$$
\begin{gathered}
f_{i}^{k}=P_{i}^{k}-\sum_{j=1}^{m} \hat{T}_{i j}^{k} \cdot x_{i}^{k} \cdot x_{j}^{k} \cong 0 \\
f_{j}^{k}=A_{j}^{k}-\sum_{i=1}^{n} \hat{T}_{i j}^{k} \cdot x_{i}^{k} \cdot x_{j}^{k} \cong 0 \\
f_{t}^{k}=\sum_{t} \hat{T}_{i j}^{k} \cdot x_{i}^{k} \cdot x_{j}^{k}-\sum_{t} T_{i j}^{k} \cong 0 \\
\sum_{k} \hat{T}_{i j}^{k} \cdot x_{i}^{k} \cdot x_{j}^{k} \leq u_{i j} \\
x_{i}^{k}, x_{j}^{k} \geq 0
\end{gathered}
$$

Obviously, the fuzzy model (15) calibrates all of the approximation functions simultaneously. Now, according to Zimmermann's approach [20], the optimal solution of the above fuzzy model can be obtained by solving eqn (16):

$$
\operatorname{Minimize}\left[\operatorname{Min}\left[\mu_{i}\left(\hat{T}_{i j}^{k}\right), \mu_{j}\left(\hat{T}_{i j}^{k}\right), \mu_{t}\left(\hat{T}_{i j}^{k}\right)\right]\right]
$$


If the membership functions are defined as follows:

$$
\mu_{j}\left(\hat{T}_{i j}^{k}\right)=\left\{\begin{array}{cc}
1+\frac{f_{j}^{k}}{r_{j}} & -r_{j} \leq f_{j}^{k} \leq 0 \\
1-\frac{f_{j}^{k}}{r_{j}} & 0 \leq f_{j}^{k} \leq r_{j} \\
0 & \text { otherwise }
\end{array}\right.
$$

where the tolerances $q_{i}, r_{j}$ and $v_{t}$ are given initially. By substituting:

$$
\operatorname{Min}\left[\mu_{j}\left(\hat{T}_{i j}^{k}\right), \mu_{i}\left(\hat{T}_{i j}^{k}\right), \mu_{t}\left(\hat{T}_{i j}^{k}\right)\right]=\lambda
$$

We get:

$$
\begin{gathered}
(\lambda-1) q_{i} \leq f_{i}^{k} \leq(1-\lambda) q_{i},(\lambda-1) r_{j} \leq f_{j}^{k} \leq(1-\lambda) r_{j}, \\
(\lambda-1) v_{t} \leq f_{t}^{k} \leq(1-\lambda) v_{t}
\end{gathered}
$$

Therefore, the proposed model (P) is obtained from eqns (15), (16) and (19):

$\operatorname{Min} \lambda$

(Model P)

st:

$$
\begin{gathered}
(\lambda-1) r_{j} \leq A_{j}^{k}-\sum_{i=1}^{n} \hat{T}_{i j}^{k}(I) \leq(1-\lambda) r_{j} \\
(\lambda-1) q_{i} \leq P_{i}^{k}-\sum_{j=1}^{m} \hat{T}_{i j}^{k}(I) \leq(1-\lambda) q_{i} \\
(\lambda-1) v_{t} \leq \sum_{t} \hat{T}_{i j}^{k}(I)-\sum_{t} T_{i j}^{k} \leq(1-\lambda) v_{t} \\
\sum_{k} \hat{T}_{i j}^{k}(I) \leq u_{i j} \\
\hat{T}_{i j}^{k}(I)=\hat{T}_{i j}^{k} \cdot x_{i}^{k} \cdot x_{j}^{k} \\
x_{i}^{k}, x_{j}^{k}, \lambda, \hat{T}_{i l}^{k}(I) \geq 0
\end{gathered}
$$

Indeed, this model (P) calibrates the approximation functions (5). Fortunately, since most of the constraints in model $(\mathrm{P})$ are of quadratic form, this model can be handled by Lingo software. It is worth mentioning that the quadratic model is convex [24]. 
STEP 4 (Forecast trip distribution): suppose that $P_{i}^{k^{\prime}}, A_{j}^{k^{\prime}}, L_{i j}^{k^{\prime}}, t_{i j}^{k^{\prime}}$ are the parameters of a multi-commodity network in the future. Trip distribution in the future $\left(T_{i j}^{k^{\prime}}\right)$ is forecasted by eqn (20):

$$
\begin{aligned}
T_{i j}^{k^{\prime}} & =f_{k}\left(P_{i}^{k^{\prime}}(s), A_{j}^{k^{\prime}}(s), L_{i j}^{k^{\prime}}(s), t_{i j}^{k^{\prime}}(s)\right) \rightarrow \\
T_{i j}^{k^{\prime}} & =\sum_{h} W_{h}^{k} G\left(\underline{x}_{i j}^{k^{\prime}}(s), \underline{X}_{[h]}^{k^{\prime}}(s)\right) \cdot x_{i}^{k^{\prime}} \cdot x_{j}^{k^{\prime}}
\end{aligned}
$$

Finally, the proposed model (F) calibrates $T_{i j}^{k^{\prime}}$ and trip distribution in the future is obtained by solving this model:

$\operatorname{Min} \lambda$

(Model F)

st:

$$
\begin{gathered}
(\lambda-1) r_{j}^{\prime} \leq A_{j}^{k^{\prime}}-\sum_{i=1}^{n} \hat{T}_{i j}^{k^{\prime}}(I) \leq(1-\lambda) r_{j}^{\prime} \\
(\lambda-1) q_{i}^{\prime} \leq{P_{i}^{k}}^{\prime}-\sum_{j=1}^{m} \hat{T}_{i j}^{{ }^{\prime}}(I) \leq(1-\lambda) q_{i}^{\prime} \\
\sum_{k} \hat{T}_{i j}^{k^{\prime}}(I) \leq u_{i j}^{\prime} \\
\hat{T}_{i j}^{k^{\prime}}(I)=\hat{T}_{i j}^{k^{\prime}} \cdot x_{i}^{k^{\prime}} \cdot x_{j}^{k} \\
x_{i}^{{ }^{\prime}}, x_{j}^{{ }^{\prime}}, \lambda, \hat{T}_{i j}^{k^{\prime}}(I) \geq 0
\end{gathered}
$$

\subsection{Dimension consideration}

If we consider the multi-commodity transportation network with $n$ nodes, $r$ arcs and $m$ commodities:

a. Maximum size of the model $P$ will be $(n+\mathrm{r}) . m$ constraints and $n . m$ variables which could be handled in the case of large scale.

b. Size of the model $F$ will be $n . m$ constraints and variables.

It is worth mentioning that the main contribution of this research is to develop two new optimization problems (P) and (F). The optimal solution of model (F) can be obtained by Lingo software. Now, the proposed model is illustrated by a numerical example.

\subsection{Numerical example}

Consider the following two-commodity network. Trips produced, trips attracted, the travel time and trip distribution at present between zones for each commodity are given in Table 1. Find trip distribution based on given data in Table 2. 


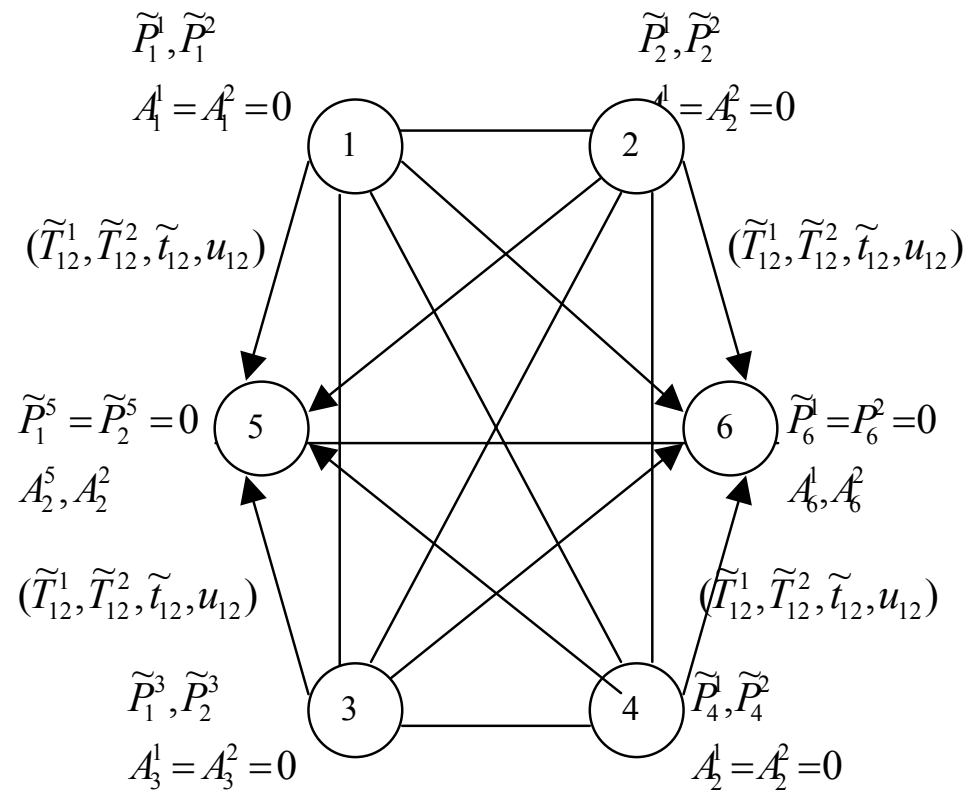

Figure 1: Two-commodity transportation network.

Table 1: $\quad$ Parameters at present (given data).

\begin{tabular}{|c|c|}
\hline$\widetilde{T}_{15}^{1}=(230,240,245,250)$ & $\widetilde{P}_{1}^{1}=(390,400,405,410)$ \\
\hline$\widetilde{T}_{15}^{2}=\left(\begin{array}{lllll}410 & , 420 & , 425 & , 430\end{array}\right)$ & $\widetilde{P}_{1}^{2}=(690,700,705,710)$ \\
\hline$\widetilde{T}_{16}^{1}=\left(\begin{array}{lllll}150 & 160 & , 165 & , 170\end{array}\right)$ & $\widetilde{P}_{2}^{1}=(490,500,505,510)$ \\
\hline$\widetilde{T}_{16}^{2}=(270,280,285,290)$ & $\widetilde{P}_{2}^{2}=(390,400 \quad, 405 \quad, 410 \quad)$ \\
\hline$\widetilde{T}_{25}^{1}=\left(\begin{array}{llll}290 & 300,305,310\end{array}\right)$ & $\widetilde{P}_{3}{ }^{1}=\left(\begin{array}{llll}490,500,505,510\end{array}\right)$ \\
\hline$\widetilde{T}_{25}^{2}=\left(\begin{array}{lllll}150 & , 160 & , 165 & , 170\end{array}\right)$ & $\widetilde{P}_{3}^{2}=(90,100,105,110)$ \\
\hline$\widetilde{T}_{23}^{2}=\left(\begin{array}{llll}190 & , 200 & , 205 & , 210\end{array}\right)$ & $\widetilde{P}_{4}^{1}=(90,100,105,110)$ \\
\hline$\widetilde{T}_{26}^{1}=(290,300,305,310)$ & $\widetilde{P}_{4}{ }^{2}=(190,200,205,210)$ \\
\hline$\widetilde{T}_{26}^{2}=\left(\begin{array}{llll}230 & , 240 & , 245 & , 250\end{array}\right)$ & $\widetilde{A}_{5}^{1}=(690,700,705,710)$ \\
\hline$\widetilde{T}_{35}^{1}=(190,200,20,210)$ & $\widetilde{A}_{5}^{2}=\left(\begin{array}{lllll}490,500 & , 505 & , 510\end{array}\right)$ \\
\hline$\widetilde{T}_{35}^{2}=(30,40,45,50)$ & $\widetilde{A}_{6}^{1}=(790,800,805,810)$ \\
\hline$\widetilde{T}_{36}^{1}=\left(\begin{array}{llll}290 & , 300 & , 305 & , 310\end{array}\right)$ & $\widetilde{A}_{6}^{2}=\left(\begin{array}{llll}890,900,905,910\end{array}\right)$ \\
\hline$\widetilde{T}_{45}^{1}=(50,60,65,70)$ & $\widetilde{T}_{36}^{2}=(50,60,65,70)$ \\
\hline$\widetilde{T}_{46}^{1}=(30,40,45,50)$ & $\widetilde{T}_{45}^{2}=(110,120,125,130)$ \\
\hline$\widetilde{t}_{13}=\widetilde{t}_{24}=(5,6,6,5,7)$ & $\widetilde{T}_{46}^{2}=(70,80,85,90)$ \\
\hline $\begin{aligned} \widetilde{t}_{12}=\widetilde{t}_{15}=\widetilde{t}_{34} & =\widetilde{t}_{26}=\widetilde{t}_{45}= \\
\widetilde{t}_{36} & =(2,3,3.5,4)\end{aligned}$ & $\begin{aligned} \widetilde{t}_{14}=\widetilde{t}_{23}=\widetilde{t}_{16} & =\widetilde{t}_{25}=\widetilde{t}_{35}= \\
\tilde{t}_{56} & =(4.5,5.5,6,6.5)\end{aligned}$ \\
\hline$\widetilde{t}_{46}=(4,5,5.5,6)$ & \\
\hline
\end{tabular}


196 Urban Transport XIII: Urban Transport and the Environment in the 21st Century

Table 2: $\quad$ Parameter in future (given data).

\begin{tabular}{|c|c|}
\hline$\widetilde{P}_{4}{ }^{\prime}=(140,150,155,160)$ & $\widetilde{P}_{1}{ }^{\prime}{ }^{\prime}=(590,600,605,610)$ \\
\hline$\widetilde{P}_{4}^{2^{\prime}}=(240,250,255,260)$ & $\widetilde{P}_{1}{ }^{\prime}=(890,900,905,910)$ \\
\hline$\tilde{A}_{5}^{1^{\prime}}=\left(\begin{array}{llll}890 & , 900,905,910\end{array}\right)$ & $\widetilde{P}_{2}^{1^{\prime}}=(590,600,605,610)$ \\
\hline$\tilde{A}_{5}^{2^{\prime}}=(690,700,705,710)$ & $\widetilde{P}_{2}^{2^{\prime}}=(490,500,505,510)$ \\
\hline$\widetilde{A}_{6}^{1{ }^{\prime \prime}}=\left(\begin{array}{lllll}940 & , 950 & , 955,960\end{array}\right)$ & $\widetilde{P}_{3}{ }^{\prime}=(490,500,505,510)$ \\
\hline$\tilde{A}_{6}^{2^{\prime}}=\left(\begin{array}{llll}1090,1100,1105 & , 110\end{array}\right)$ & $\widetilde{P}_{3}^{2^{\prime}}=(140,150,155,160)$ \\
\hline$\tilde{t}_{56}^{\prime}=(4,5,5.5,6)$ & $\tilde{t}_{13}^{\prime}=\tilde{t}_{24}^{\prime}=(4.5,5.5,6,6.5)$ \\
\hline $\begin{array}{r}\tilde{t}_{14}^{\prime}=\tilde{t}_{23}{ }^{\prime}=\tilde{t}_{16}{ }^{\prime}=\widetilde{t}_{25}{ }^{\prime}=\widetilde{t}_{35}{ }^{\prime}= \\
\tilde{t}_{46}^{\prime}=(3,4,4.5,5)\end{array}$ & $\begin{array}{r}\tilde{t}_{12}{ }^{\prime}=\tilde{t}_{15}{ }^{\prime}=\tilde{t}_{34}{ }^{\prime}=\tilde{t}_{26}{ }^{\prime}=\tilde{t}_{45}{ }^{\prime}= \\
\tilde{t}_{36}{ }^{\prime}=(2.4,2.5,2.55,2.6)\end{array}$ \\
\hline
\end{tabular}

$\operatorname{Min} \lambda$

(Model F)

st:

$$
\begin{aligned}
& -5(1-\lambda) \leq 600-\left(\hat{T}_{15}^{1}{ }^{\prime}(I)+\hat{T}_{16}^{1}{ }^{\prime}(I)\right) \leq 5(1-\lambda) \\
& -5(1-\lambda) \leq 900-\left(\hat{T}_{15}^{2}{ }^{\prime}(I)+\hat{T}_{16}^{2}{ }^{\prime}(I)\right) \leq 5(1-\lambda) \\
& -5(1-\lambda) \leq 600-\left(\hat{T}_{25}{ }^{\prime}(I)+\hat{T}_{26}{ }^{\prime}(I)\right) \leq 5(1-\lambda) \\
& -5(1-\lambda) \leq 500-\left(\hat{T}_{25}{ }^{\prime}(I)+\hat{T}_{26}{ }^{\prime}(I)\right) \leq 5(1-\lambda) \\
& -5(1-\lambda) \leq 500-\left(\hat{T}_{35}{ }^{\prime}(I)+\hat{T}_{36}{ }^{\prime}(I)\right) \leq 5(1-\lambda) \\
& -5(1-\lambda) \leq 150-\left(\hat{T}_{35}^{2}{ }^{\prime}(I)+\hat{T}_{36}^{2}{ }^{\prime}(I)\right) \leq 5(1-\lambda) \\
& -5(1-\lambda) \leq 150-\left(\hat{T}_{45}^{1}{ }^{\prime}(I)+\hat{T}_{46}^{1}{ }^{\prime}(I)\right) \leq 5(1-\lambda) \\
& -5(1-\lambda) \leq 250-\left(\hat{T}_{45}^{2}(I)+\hat{T}_{46}^{2}(I)\right) \leq 5(1-\lambda) \\
& -5(1-\lambda) \leq 900-\left(\hat{T}_{15}^{1}{ }^{\prime}(I)+\hat{T}_{45}^{1}{ }^{\prime}(I)+\hat{T}_{25}{ }^{\prime}(I)+\hat{T}_{35}{ }^{\prime}(I)\right) \leq 5(1-\lambda) \\
& -5(1-\lambda) \leq 700-\left(\hat{T}_{15}^{2{ }^{\prime}}(I)+\hat{T}_{45}^{2{ }^{\prime}}(I)+\hat{T}_{25}^{2{ }^{\prime}}(I)+\hat{T}_{35}^{2{ }^{\prime}}(I)\right) \leq 5(1-\lambda) \\
& -5(1-\lambda) \leq 950-\left(\hat{T}_{26}^{1}{ }^{\prime}(I)+\hat{T}_{36}{ }^{\prime}{ }^{\prime}(I)+\hat{T}_{16}{ }^{\prime}{ }^{\prime}(I)+\hat{T}_{46}^{1}{ }^{\prime}(I)\right) \leq 5(1-\lambda) \\
& -5(1-\lambda) \leq 1100-\left(\hat{T}_{26}^{2}{ }^{\prime}(I)+\hat{T}_{36}{ }^{\prime}(I)+\hat{T}_{16}^{2{ }^{\prime}}(I)+\hat{T}_{46}^{2}(I)\right) \leq 5(1-\lambda) \\
& \hat{T}_{15}^{1{ }^{\prime}}(I)=318.709 x_{1}^{1^{\prime}} \cdot x_{5}^{1^{\prime}} \quad \hat{T}_{15}^{{ }^{\prime}}(I)=318.709 x_{1}^{1^{\prime}} \cdot x_{5}^{1^{\prime}} \\
& \hat{T}_{16}{ }^{\prime}{ }^{\prime}(I)=263.204 x_{1}^{1^{\prime}} \cdot x_{6}^{1^{\prime}} \quad \hat{T}_{16}{ }^{\prime}{ }^{\prime}(I)=263.204 x_{1}^{1^{\prime}} \cdot x_{6}^{1^{\prime}} \\
& \hat{T}_{25}{ }^{\prime}{ }^{\prime}(I)=281.775 x_{2}^{1^{\prime}} \cdot x_{5}^{1^{\prime}} \quad \hat{T}_{25}^{1{ }^{\prime}}(I)=281.775 x_{2}^{1^{\prime}} \cdot x_{5}^{1^{\prime}} \\
& \hat{T}_{26}{ }^{\prime}{ }^{\prime}(I)=300.404 x_{2}^{1^{\prime}} \cdot x_{6}^{1^{\prime}} \quad \hat{T}_{26}{ }^{\prime}{ }^{\prime}(I)=300.404 x_{2}^{1^{\prime}} \cdot x_{6}^{1^{\prime}} \\
& \hat{T}_{35}{ }^{\prime}{ }^{\prime}(I)=235.419 x_{3}^{1^{\prime}} \cdot x_{5}^{1^{\prime}} \quad \hat{T}_{35}{ }^{\prime}{ }^{\prime}(I)=235.419 x_{3}^{1^{\prime}} \cdot x_{5}^{1^{\prime}} \\
& \hat{T}_{36}{ }^{\prime}{ }^{\prime}(I)=256.766 x_{3}^{1^{\prime}} \cdot x_{6}^{1^{\prime}} \quad \hat{T}_{36}{ }^{\prime}{ }^{\prime}(I)=256.766 x_{3}^{1^{\prime}} \cdot x_{6}^{1^{\prime}} \\
& \hat{T}_{45}^{1}{ }^{\prime}(I)=69.095 x_{4}^{1^{\prime}} \cdot x_{5}^{1^{\prime}} \quad \hat{T}_{45}{ }^{\prime}(I)=69.095 x_{4}^{1^{\prime}} \cdot x_{5}^{1^{\prime}} \\
& \hat{T}_{46}{ }^{\prime}{ }^{\prime}(I)=53.881 x_{4}^{1^{\prime}} \cdot x_{6}^{1^{\prime}} \quad \hat{T}_{46}{ }^{\prime}{ }^{\prime}(I)=53.881 x_{4}^{1^{\prime}} \cdot x_{6}^{1^{\prime}} \\
& \hat{T}_{15}^{2 \prime}(I), \hat{T}_{16}^{2{ }^{\prime}}(I), \hat{T}_{25}^{2{ }^{\prime}}(I), \hat{T}_{26}{ }^{\prime}{ }^{\prime}(I), \hat{T}_{35}^{2{ }^{\prime}}(I), \hat{T}_{36}{ }^{\prime}{ }^{\prime}(I), \hat{T}_{45}{ }^{\prime}{ }^{\prime}(I), \hat{T}_{46}^{2{ }^{\prime}}(I) \geq 0
\end{aligned}
$$


The optimal solution of model (F) is given in Table 3 (solved by Lingo software).

Table 3: $\quad$ The optimal solution.

\begin{tabular}{|c|c|c|c|c|c|c|}
\hline \multicolumn{4}{|c|}{ New Model } & \multicolumn{2}{|c|}{ Survey model } & \multirow{3}{*}{ 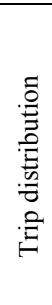 } \\
\hline \multicolumn{2}{|c|}{ Forecasting } & \multicolumn{2}{|c|}{ Present } & \multicolumn{2}{|c|}{ Present } & \\
\hline 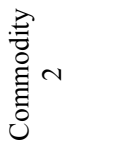 & 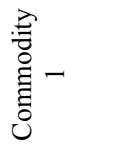 & 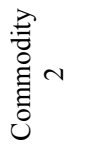 & 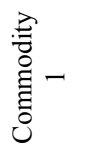 & 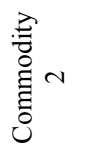 & 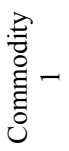 & \\
\hline 404.3265 & 310.5853 & 420 & 240 & 420 & 240 & T15 \\
\hline 490.6735 & 284.4147 & 280 & 160 & 280 & 160 & T16 \\
\hline 145.1731 & 277.2482 & 160 & 200 & 160 & 200 & $\mathrm{~T} 25$ \\
\hline 359.8269 & 327.7518 & 240 & 300 & 240 & 300 & T26 \\
\hline 44.47258 & 224.0430 & 40 & 200 & 40 & 200 & T35 \\
\hline 110.5274 & 270.9570 & 60 & 300 & 60 & 300 & T36 \\
\hline 111.0278 & 83.12359 & 120 & 60 & 120 & 60 & T45 \\
\hline 133.9722 & 71.87641 & 80 & 40 & 80 & 40 & T46 \\
\hline
\end{tabular}

By referring to Table 3, we could see the forecasted trip distribution between the zones for some commodities gets higher (e.g. T16 for commodity 2 which is 280 at present rises to 490.67 in the future). If we consider the Table 3 in more detail, we could find similar cases. This trip distribution forecasting identifies that the volume of traffic will exceed the capacity of paths. Thus, capacity planning and traffic engineering are required.

\section{Conclusions}

The author has presented a model to learn the present state of real trip distribution by RBF neural network. In addition, a novel model has been introduced to forecast the trip distribution in multi-commodity transportation networks in both crisp and fuzzy environments. This new approach is constructed based on a fuzzy multi-objective optimization model, which can be solved easily using Lingo software. As an advantage, the model incorporates all calibrations simultaneously to save the execution time. On the other hand, the special structure of the proposed model is very close to quadratic programming where its convexity is confirmed.

A numerical example has been then presented to demonstrate the accuracy of solutions gained for the present state of trip distribution from the proposed 
model. The efficiency of this method has been indicated using dimension consideration for large-scale transportation networks.

Modeling data distribution on intelligent multi-commodity transportation networks is the subject of further research.

\section{References}

[1] Low, D.A., A New Approach to Transportation System Modeling, Traffic Quarterly, 26(3), 1972.

[2] Holm, Jesen, T., Nielsen, S.K., Christensen, A., Johnsen, B. and Ronby, G., Calibrating Traffic Models on Traffic Census Results Only, Traffic Engineering and Control, pp. 137-140, 1976.

[3] Gaudry, M. and Lamarre, L., Estimating Origin-Destination Matrices from Traffic Counts: A Simple Linear Intercity Model for Quebec, No. 105, Center for Transportation Research, University of Montreal, 1978.

[4] Robillard, P., Estimation the O-D Matrix from Observed Link Volumes, Transportation Research, 9, pp. 123-128, 1975.

[5] Hogberg, P., Estimation of Parameters in Models for Traffic Prediction: A Non-Linear Regression Approach, Transportation Research, 10, pp. $263-$ 265, 1976.

[6] Williumsen, L.G., Estimation of an O-D Matrix from Traffic Counts- A Review, Institute of Transportation Studies, University of Leeds, England, 1978.

[7] Van Zuylen, H.J., The Information Minimization Method: Validity Applicability to Transport Planning, New Developments in Modeling Travel Demand and Urban Systems, ed. G.R.H. Jansen et al., Saxon, Farnborough, 1978.

[8] Van Zuylen, H.J., The Estimation of Turning Flows on a Junction, Traffic Engineering and Control, 20(11), pp. 539-541, 1979.

[9] McNeil, S. and Hendrickson, C., A Regression Formulation on Matrix Estimation, Transportation Science, 19(3), pp. 278-292, 1983.

[10] Geva, I. and Hauerlandau, E., Maximum-Likelihood and Bayesian Methods for the Estimation of Origin-Destination Flows, Transportation Research Record, No. 944, pp. 101-105, 1983.

[11] Spiess, H., A Maximum Likelihood Model for Estimating OriginDestination Matrices, Transportation Research, 21B, pp. 395-412, 1987.

[12] Walting, D.P. and Maher, M.J., A Statistical Procedure for Estimating a Mean Origin-Destination Matrix from a Partial Registration Plate Survey, Transportation Research, 26B, pp. 171-193, 1992.

[13] Walting, D.P. and Grey, D.R., Analysis of Partial Registration Plate Data Using a Model with Poisson Input and Output, Proc. of the Int. Conf. on Mathematics in Transport Planning and Control, Oxford University Press, 1991.

[14] Kalic, M. and Teodorovic, D., Trip Distribution Modeling Using Soft Computing Techniques, EURO XV/ INFORMS XXXIV, pp. 74, Barcelona, Spain, 1997. 
[15] Mozolin, M., Thill, J.C. and Usery, E.L., Trip Distribution Forecasting with Multi-Layer Perceptron Neural Networks: A Critical Evaluation, Transportation Research B, 34, pp. 53-73, 2000.

[16] Kalic, M. and Teodorović, D., Trip Distribution Using Fuzzy Logic and Genetic Algorithm, Transportation Planning and Technology, 26, pp. 213-238, 2003.

[17] Bazaraa, S., Sherali, D. and Jarvis, J., Linear Programming and Network Flows, 3rd Edition, John Wiley \& Sons, 2004.

[18] Khisty, C., and Lall, B. Transportation Engineering: An Introduction, 3rd Edition, Prentice-Hall International, August 2002.

[19] Haykin, S., Neural Networks: a Comprehensive Foundation, $2^{\text {nd }}$ Edition, New Jersey, Prentice Hall, 1998.

[20] Zimmerman, Fuzzy Set Theory and Its Application, Kluwer Academic Publisher, 1993.

[21] Bazaraa, S., Sherali, H.D. and Shetty, C.M., Nonlinear Programming: Theory and Algorithms, 2nd Edition, John Wiley Sons, 1993.

[22] Hwang, C.L., Fuzzy Mathematical Programming Methods and Application, Springer-Verlag, 1989.

[23] Masud, H. Multi Objective Decision Making, Methods and Application, Springer-Verlag, 1989.

[24] K. G. Murty, Linear programming, John Wiley Sons, 1983. 